L. da Silva, M. Spite, J. R. de Medeiros, eds.

\title{
Light Element Evolution at the Solar Neighborhood
}

\author{
Andreu Alibés, Javier Labay and Ramon Canal \\ Departament d'Astronomia $i$ Meteorologia, Universitat de Barcelona, \\ C/Martí i Franquès 1, 08028 Barcelona, Spain
}

\begin{abstract}
We present the Light Element Evolution resulting from our new Chemical Evolution model. The LiBeB evolution is correctly fitted by taking into account several sources: Big Bang, Galactic Cosmic Ray Nucleosynthesis, the $\nu$-process, novae and AGB and C-stars.
\end{abstract}

\section{Introduction}

Our aim in this work has been to study the synthesis and evolution of the abundances of the light elements in the Solar Neighborhood from the birth of the Galaxy to present. Light elements have sources different from those of heavier elements. We have included GCR nucleosynthesis in a complete Galactic Chemical Evolution Model that takes into account 76 stable isotopes from hydrogen to zinc. Any successful $\mathrm{LiBeB}$ evolution model should also be compatible with other observational constraints like the age-metallicity relation, the G-dwarf distribution or the evolution of other elements. Some of the standard outputs of our chemical evolution model, which is in good agreement with those observational data, are the helium, carbon, nitrogen and oxygen evolution in the ISM. Those elements are the targets of the GCR made of the newly synthesized material in a type II supernova, accelerated by the shock wave.

\section{Sources considered}

We have considered several stellar and interstellar frameworks for the production of the light elements. The main source of ${ }^{6} \mathrm{Li},{ }^{9} \mathrm{Be}$ and ${ }^{10} \mathrm{~B}$ is the Galactic Cosmic Ray Nucleosynthesis, due to spallation reactions between Cosmic Ray particles and ISM material. Following Ramaty et al. (1997), and using their LIBEB code, we have calculated the production rate by this mechanism, resulting from the ISM abundances calculated by our model being hit by GCR composed of material newly synthesized and ejected by a SNII. The source energy spectrum of the GCR is: $q(E) \propto \frac{p^{-2.2}}{\beta} e^{-\frac{E}{E_{0}}}$, with $E_{0}=10 \mathrm{GeV} / \mathrm{n}$.

Neutrino induced nucleosynthesis. Light elements are produced when the strong neutrino flux produced in a core collapse supernova goes through the material surrounding the core. Its yields, which depend on that neutrino flux, are quite uncertain. This mechanism produces ${ }^{7} \mathrm{Li}$ and ${ }^{11} \mathrm{~B}$ and we have fixed the contribution of Woosley and Weaver $(1995)$ yields by means of $\left({ }^{11} \mathrm{~B} /{ }^{10} \mathrm{~B}\right)_{\odot}$. 

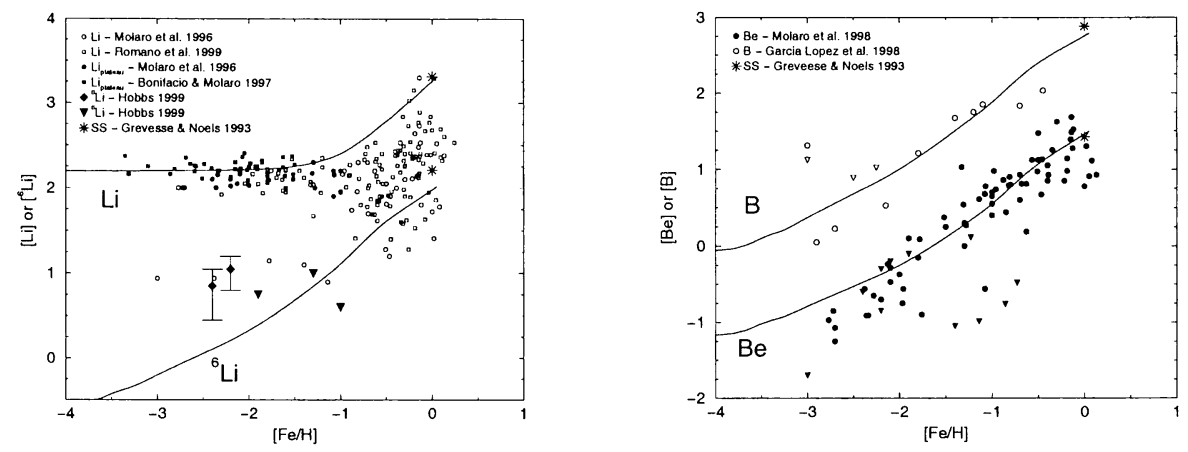

Novae are supposed to produce some ${ }^{7} \mathrm{Li}$ in each outburst. We calculate the outburst rate following the equation: $D \int_{m(t)+0.5}^{9.5} \frac{\phi\left(m_{B}\right)}{m_{B}} \int_{\mu_{m}}^{\mu_{M}} f(\mu) \psi\left(t-\tau_{m_{B}(1-\mu)}-\right.$ $t_{\text {cool }}$ ) where $D$ imposes the actual outburst rate and $t_{\text {cool }}=1 \mathrm{Gyr}$ is the cooling time for a white dwarf until it can produce the first nova outburst. The nova system we have considered is made of a white dwarf coming from an initial 1-8 $\mathrm{M}_{\odot}$ primary star and a $0.5-1.5 \mathrm{M}_{\odot}$ secondary star. Each outburst ejects on average $1.03 \cdot 10^{-10} \mathrm{M}_{\odot}$ of ${ }^{7} \mathrm{Li}$ (José \& Hernanz 1998).

$A G B$ and $C$-stars are also ${ }^{7} \mathrm{Li}$ producers. We have included them as in Abia et al. 1993, using a present production rate of $P_{7}=10^{-8} \mathrm{M}_{\odot} \mathrm{pc}^{-2} \mathrm{Gyr}^{-1}$, in order to fit the lithium evolution. That rate is just 5 times larger than the one statistically determined by those authors, but lower than the one needed in their evolution model.

The results are shown in the figures.
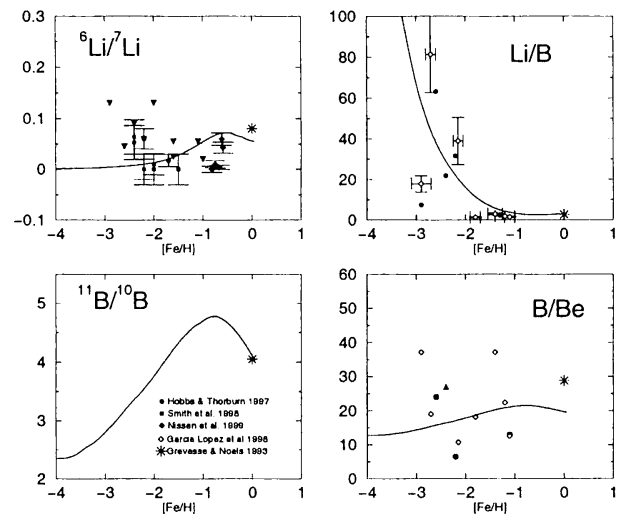

\section{References}

Abia, C., Isern, J., \& Canal, R. 1993, A\&A, 275, 96

Chiappini, C., Matteucci, F., \& Gratton, R. 1997, ApJ, 477, 765

José, J., \& Hernanz, M. 1998, ApJ, 494, 680

Matteucci, F., D'Antona, F., \& Timmes, F.X. 1995, A\&A, 303, 460

Ramaty, R., Kozlovsky, B., Lingenfelter, R.E., \& Reeves, H. 1997, ApJ, 488, 730

Romano, D., Matteucci, F., Molaro, P., \& Bonifacio, P. 1999, A\&A, 352, 117

Woosley, S.E., \& Weaver, T.A. 1995, ApJS, 101, 181 